\title{
Pentacyclic Triterpenoids from the Aerial Parts of Lantana camara
}

\author{
Sabira Begum, * Aneela WaHAB, and Bina Shaheen SiddiQui \\ H.E.J. Research Institute of Chemistry, International Center for Chemical Sciences, University of Karachi; Karachi 75270, \\ Pakistan. Received August 23, 2002; accepted October 23, 2002
}

\begin{abstract}
Three new pentacyclic triterpenoids, camaryolic acid (1), methylcamaralate (2) and camangeloyl acid (3) and six known compounds $\beta$-sitosterol 3- $O$ - $\beta$-D-glucopyranoside (4), octadecanoic acid (5), docosanoic acid (6), palmitic acid (7), camaric acid (8) and lantanolic acid (9) were isolated from the aerial parts of Lantana camara. Structures of the new compounds were elucidated by spectroscopic and chemical methods.
\end{abstract}

Key words Lantana camara; Verbenaceae; pentacyclic triterpenoid; camaryolic acid; methylcamaralate; camangeloyl acid

Lantana camara L. (Verbenaceae) is a hairy shrub, native to tropical America. Different parts of this plant are used for the treatment of various human ailments such as itches, cuts, ulcers, swellings, bilious fever, catarrh, eczema, tetanus, malaria, tumors and rheumatism. ${ }^{1,2)}$ Phytochemical studies carried out by different groups of workers on different parts of the plant have resulted in the isolation of various terpenoids, steroids and flavonoids. ${ }^{3-5)}$ In the course of investigations on the constituents of the aerial parts of L. camara, three new pentacyclic triterpenoids namely camaryolic acid (1), methylcamaralate (2) and camangeloyl acid (3) were isolated. The structures of these constituents were elucidated as 3,25-epoxy-3 $\alpha$-methoxy-22 $\beta$-[ $\beta, \beta$-dimethylacryloyloxy]urs-12-en-28-oic acid, methyl $22 \beta$-acetoxy-3,25-epoxy-3 $\alpha$ hydroxy-urs-12-en-28-oate and 3,25-epoxy-3 $\alpha$-hydroxy- $22 \beta$ $\left[(Z)-2^{\prime}\right.$-methyl-2'-butenoyloxy]-11-oxoolean-12-en-28-oic acid respectively on the basis of various 2D-NMR techniques including ${ }^{1} \mathrm{H}-{ }^{1} \mathrm{H}$ correlation spectroscopy (COSY), nuclear overhauser enhancement spectroscopy (NOESY), heteronuclear multiple quantum coherence (HMQC) and heteronuclear multiple bond connectivity (HMBC). In addition six known compounds $\beta$-sitosterol 3- $O$ - $\beta$-D-glucopyranoside (4), ${ }^{6}$ octadecanoic acid (5), ${ }^{7}$ docosanoic acid (6), ${ }^{8)}$ palmitic acid (7), ${ }^{9)}$ camaric acid $(\mathbf{8})^{10)}$ and lantanolic acid $(\mathbf{9})^{11)}$ were isolated from this plant. This is the first report of the isolation of compound $\mathbf{4}$ from $L$. camara. Herein, we report the structural assignments of the three new triterpenoids.

\section{Results and Discussion}

The high resolution electron impact mass spectrum (HREI-MS) of 1 showed the $\mathrm{M}^{+}$peak at $m / z 582.3918$ corresponding to the molecular formula $\mathrm{C}_{36} \mathrm{H}_{54} \mathrm{O}_{6}$. Its infrared (IR) spectrum showed absorption bands at 3450-2620 (br, $\mathrm{COOH}$ ), 1730 (ester carbonyl), 1710 (acid carbonyl) and $1620 \mathrm{~cm}^{-1}$ (olefinic double bond). The ${ }^{1} \mathrm{H}-\mathrm{NMR}$ spectrum (Table 1) of 1 showed eight methyl signals, four tertiary methyls as singlets $\left(\delta_{\mathrm{H}} 0.74,0.95,1.00\right.$ and 1.05) and two secondary methyls as doublets at $\delta_{\mathrm{H}} 0.85(J=6.2 \mathrm{~Hz})$ and $\delta_{\mathrm{H}}$ $0.89(J=6.4 \mathrm{~Hz})$ and two tertiary methyl signals on the double bond. It also exhibited a one-proton doublet at $\delta_{\mathrm{H}} 2.40$ $(J=11.2 \mathrm{~Hz})$. These data showed that $\mathbf{1}$ belongs to the $\alpha$ amyrin series of pentacyclic triterpenoids, which was confirmed by observing the characteristic ${ }^{12)}$ signals in the ${ }^{13} \mathrm{C}$ NMR spectrum (Table 2) at $\delta_{\mathrm{C}} 125.9(\mathrm{C}-12)$ and $\delta_{\mathrm{C}} 138.2$ (C-13). Two one-proton double doublets appeared at $\delta_{\mathrm{H}} 4.26$ $(J=8.9,2.7 \mathrm{~Hz})$ and $\delta_{\mathrm{H}} 3.88(J=8.9,1.0 \mathrm{~Hz})$ due to two nonequivalent methylene protons $\mathrm{H}-25 \mathrm{a}$ and $\mathrm{H}-25 \mathrm{~b}\left(\delta_{\mathrm{C}} 67.7\right.$; distortionless enhancement by polarization transfer (DEPT) and HMQC) respectively. Two one-proton triplets at $\delta_{\mathrm{H}} 5.00$ $(J=3.0 \mathrm{~Hz})\left(\delta_{\mathrm{C}} 75.3\right.$; DEPT and HMQC $)$ and $\delta_{\mathrm{H}} 5.23$ $(J=3.6 \mathrm{~Hz})\left(\delta_{\mathrm{C}} 125.9\right.$; DEPT and HMQC) were ascribed to $\mathrm{H}-22 \alpha$ and $\mathrm{H}-12$ respectively indicating the presence of $\beta$-oriented ester side chain at $\mathrm{C}-22$ and a double bond at position 12 . It formed methyl ester $\left(1 \mathrm{a}, \delta_{\mathrm{H}} 3.50 \mathrm{~s}\right)$ on treatment with diazomethane. Retro-Diels-Alder fragmentation ${ }^{13)}$ around ring $\mathrm{C}$ followed by the loss of the ester side chain in the form of acid, resulted in an ion at $\mathrm{m} / \mathrm{z} 246.1599$ $\left(\mathrm{C}_{16} \mathrm{H}_{22} \mathrm{O}_{2}\right)$ indicating the presence of $\mathrm{COOH}$ group at $\mathrm{C}-14$ or C-17. It was placed at $\mathrm{C}-17$ on the basis of ${ }^{13} \mathrm{C}$-NMR values of ring $\mathrm{E}$ which were comparable with those of compounds having similar structures. ${ }^{14)}$ Further evidence for placing the $\mathrm{COOH}$ group at $\mathrm{C}-17$ was provided by the fact that the presence of carboxyl group at $\mathrm{C}-14^{15}$ ) has a marked effect on the chemical shifts of $\mathrm{C}-12, \mathrm{C}-13$ and $\mathrm{C}-14$ i.e. ${ }^{13} \mathrm{C}$ NMR values of C-12 and C-14 appear downfield and that of $\mathrm{C}-13$ appear upfield as compared to the compounds having $\mathrm{COOH}$ group at $\mathrm{C}-17{ }^{14)}$

An OMe group which appeared at $\delta_{\mathrm{H}} 3.23\left(\delta_{\mathrm{C}} 49.3\right.$; DEPT and HMQC) as a three proton singlet in the ${ }^{1} \mathrm{H}-\mathrm{NMR}$ spectrum was located at C-3 with $\alpha$-orientation, since the compound had no further carbinylic proton in the ${ }^{1} \mathrm{H}-\mathrm{NMR}$ spectrum and the OMe showed interaction with $\mathrm{C}-3\left(\delta_{\mathrm{C}} 100.5\right)$ in the HMBC spectrum (Fig. 1). These data showed a close similarity of 1 with camaracinic acid, ${ }^{14)}$ i.e. a $\beta$-oriented ketal system at $\mathrm{C}-3$ with $\mathrm{C}-25$, a double bond at $\mathrm{C}-12$, a $\beta$-oriented ester side chain at $\mathrm{C}-22$ and a $\mathrm{COOH}$ group at $\mathrm{C}-17$ in the $\alpha$ amyrin skeleton. The mass spectrum of 1 also showed a peak at $\mathrm{m} / \mathrm{z} 83.0484$, corresponding to $\mathrm{C}_{5} \mathrm{H}_{7} \mathrm{O}$ and a peak at $\mathrm{m} / z$ $482.3372\left(\mathrm{C}_{31} \mathrm{H}_{46} \mathrm{O}_{4}\right)$, resulting from the loss of 100 mass units from the molecular ion. These observations were indicative of a dimethylacrylic ester-side chain at C-22. Comparison of ${ }^{1} \mathrm{H}-\mathrm{NMR}$ spectrum of $\mathbf{1}$ with that of lantanilic acid ${ }^{16)}$ indicated that the ester moiety of $\mathbf{1}$ was similar to that of lantanilic acid as it showed two three-proton singlets at $\delta_{\mathrm{H}}$ 1.82 and $\delta_{\mathrm{H}} 2.10$ ascribed to $\mathrm{H}-4^{\prime}$ and $\mathrm{H}-5^{\prime}$ respectively and a one-proton singlet at $\delta_{\mathrm{H}} 5.54$ for $\mathrm{H}-2^{\prime}$ suggesting a $\beta, \beta$ dimethylacrylic acid ester which was also confirmed by ${ }^{13} \mathrm{C}-\mathrm{NMR}$ values assigned to this moiety (Table 2) through HMQC and HMBC interactions and DEPT experiment. Interaction of $\mathrm{H}-22$ with $\mathrm{C}-\mathrm{1}^{\prime}$ in the HMBC spectrum confirmed this ester moiety at $\mathrm{C}-22$. The above spectral data characterized 1 as 3,25-epoxy-3 $\alpha$-methoxy- $22 \beta$ - $[\beta, \beta$ dimethylacryloyloxy]-urs-12-en-28-oic acid.

Compound 2 showed the molecular ion peak at $\mathrm{m} / \mathrm{z}$ 
Table 1. ${ }^{1} \mathrm{H}-\mathrm{NMR}$ Data $(\delta / \mathrm{ppm})$ of $\mathbf{1}-\mathbf{3}^{a}$

\begin{tabular}{|c|c|c|c|}
\hline Proton & $\mathbf{1}^{b)}$ & $2^{b)}$ & $3^{b)}$ \\
\hline \multirow[t]{2}{*}{1} & $2.10(\mathrm{~m})$ & $2.00(\mathrm{~m})$ & $2.10(\mathrm{~m})$ \\
\hline & $1.20(\mathrm{~m})$ & $1.21(\mathrm{~m})$ & $1.20(\mathrm{~m})$ \\
\hline \multirow[t]{2}{*}{2} & $1.41(\mathrm{~m})$ & $1.40(\mathrm{~m})$ & $1.50(\mathrm{~m})$ \\
\hline & $1.19(\mathrm{~m})$ & $1.18(\mathrm{~m})$ & $1.32(\mathrm{~m})$ \\
\hline 5 & $1.19(\mathrm{~m})$ & $1.19(\mathrm{~m})$ & $1.15(\mathrm{~m})$ \\
\hline 6 & $1.51(\mathrm{~m})$ & $1.50(\mathrm{~m})$ & $1.50(\mathrm{~m})$ \\
\hline 7 & $1.38(\mathrm{~m})$ & $1.37(\mathrm{~m})$ & $1.37(\mathrm{~m})$ \\
\hline 9 & $1.65(\mathrm{~m})$ & $1.68(\mathrm{~m})$ & $2.46(\mathrm{~s})$ \\
\hline \multirow[t]{2}{*}{11} & $2.12(\mathrm{~m})$ & $2.14(\mathrm{~m})$ & - \\
\hline & $1.75(\mathrm{~m})$ & $1.70(\mathrm{~m})$ & - \\
\hline 12 & $5.23(\mathrm{t}, 3.6)$ & $5.29(\mathrm{t}, 3.6)$ & $5.74(\mathrm{~s})$ \\
\hline \multirow[t]{2}{*}{15} & $2.14(\mathrm{~m})$ & $2.15(\mathrm{~m})$ & $2.14(\mathrm{~m})$ \\
\hline & $1.70(\mathrm{~m})$ & $1.72(\mathrm{~m})$ & $1.72(\mathrm{~m})$ \\
\hline 16 & $1.85(\mathrm{~m})$ & $1.87(\mathrm{~m})$ & $1.87(\mathrm{~m})$ \\
\hline 18 & $2.40(\mathrm{~d}, 11.2)$ & $2.41(\mathrm{~d}, 10.7)$ & $3.20(\mathrm{dd}, 14.0,4.2)$ \\
\hline \multirow[t]{2}{*}{19} & $1.31(\mathrm{~m})$ & $1.30(\mathrm{~m})$ & $1.70(\mathrm{~m})$ \\
\hline & & & $1.30(\mathrm{~m})$ \\
\hline 20 & $0.96(\mathrm{~m})$ & $0.95(\mathrm{~m})$ & - \\
\hline \multirow[t]{2}{*}{21} & $1.74(\mathrm{~m})$ & $1.72(\mathrm{~m})$ & $1.80(\mathrm{~m})$ \\
\hline & & & $1.50(\mathrm{~m})$ \\
\hline 22 & $5.00(\mathrm{t}, 3.0)(\alpha)$ & $5.04(\mathrm{t}, 3.2)(\alpha)$ & $5.12(\mathrm{t}, 3.0)(\alpha)$ \\
\hline 23 & $1.00(\mathrm{~s})$ & $1.02(\mathrm{~s})$ & $1.02(\mathrm{~s})$ \\
\hline 24 & $0.74(\mathrm{~s})$ & $0.73(\mathrm{~s})$ & $0.71(\mathrm{~s})$ \\
\hline \multirow[t]{2}{*}{25} & $4.26(\mathrm{dd}, 8.9,2.7)$ & $4.19(\mathrm{dd}, 8.4,2.9)$ & $4.50(\mathrm{dd}, 8.3,2.2)$ \\
\hline & $3.88(\mathrm{dd}, 8.9,1.0)$ & $3.85(\mathrm{dd}, 8.4,1.0)$ & $4.02(\mathrm{dd}, 8.3,1.1)$ \\
\hline 26 & $0.95(\mathrm{~s})$ & $0.93(\mathrm{~s})$ & $0.90(\mathrm{~s})$ \\
\hline 27 & $1.05(\mathrm{~s})$ & $1.06(\mathrm{~s})$ & $1.36(\mathrm{~s})$ \\
\hline 29 & $0.85(\mathrm{~d}, 6.2)$ & $0.86(\mathrm{~d}, 6.1)$ & $0.88(\mathrm{~s})$ \\
\hline 30 & $0.89(\mathrm{~d}, 6.4)$ & $0.88(\mathrm{~d}, 6.1)$ & $1.14(\mathrm{~s})$ \\
\hline $2^{\prime}$ & $5.54(\mathrm{~s})$ & $1.98(\mathrm{~s})$ & - \\
\hline $3^{\prime}$ & - & - & $6.01(\mathrm{qq}, 7.2,1.5)$ \\
\hline $4^{\prime}$ & $1.82(\mathrm{~s})$ & - & $1.94(\mathrm{dq}, 7.2,1.5)$ \\
\hline $5^{\prime}$ & $2.10(\mathrm{~s})$ & - & 1.79 (quintet, 1.5) \\
\hline $3-\mathrm{OCH}_{3}$ & $3.23(\mathrm{~s})$ & - & - \\
\hline $28-\mathrm{OCH}_{3}$ & - & $3.46(\mathrm{~s})$ & - \\
\hline
\end{tabular}

a) $400 \mathrm{MHz}$ in $\mathrm{CDCl}_{3}$. b) Figures in parentheses denote $J$ values $(\mathrm{Hz}) . \delta$ in ppm from TMS.

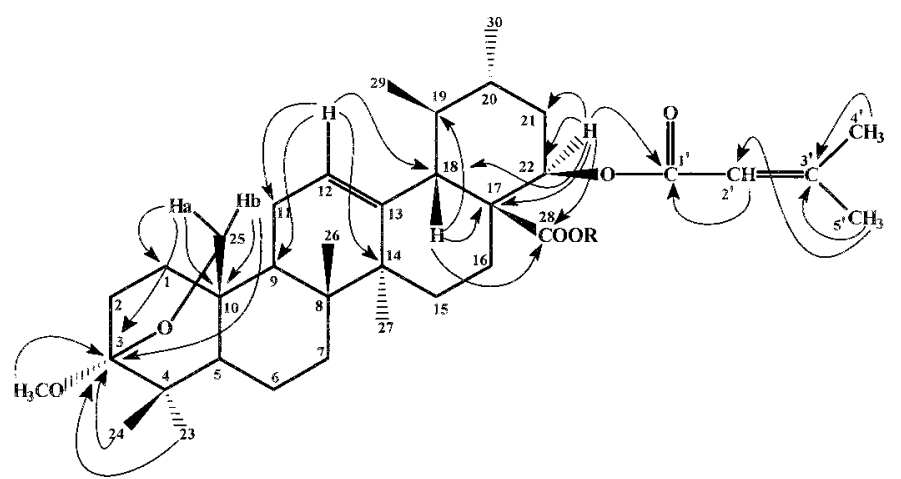

Fig. 1. Significant $\mathrm{HMBC}$ Interactions of $\mathbf{1} \mathrm{R}=\mathrm{H}$ and $\mathbf{1 a} \mathrm{R}=\mathrm{CH}_{3}$

542.3900 in the HR-EI-MS corresponding to the molecular formula $\mathrm{C}_{33} \mathrm{H}_{50} \mathrm{O}_{6}$. Its IR spectrum showed absorption bands at $3450(\mathrm{OH}), 1740($ ester $\mathrm{C}=\mathrm{O})$ and $1620 \mathrm{~cm}^{-1}(\mathrm{C}=\mathrm{C})$. Analysis of the ${ }^{1} \mathrm{H}$ - and ${ }^{13} \mathrm{C}-\mathrm{NMR}$ spectra (Table 1,2) indicated that its structure is similar to that of compound 1 except that it has acetoxy group at $\mathrm{C}-22\left(\delta_{\mathrm{H}} 1.98,3 \mathrm{H}, \mathrm{s} ; \delta_{\mathrm{C}}\right.$ $\left.170.0\left(\mathrm{C}-1^{\prime}\right), 20.7\left(\mathrm{C}-2^{\prime}\right)\right)$ instead of acryloxy side chain. Further, its ${ }^{1} \mathrm{H}-\mathrm{NMR}$ spectrum also showed the presence of OMe group $\left(\delta_{\mathrm{H}} 3.46, \mathrm{~s} ; \delta_{\mathrm{C}} 51.2 ;\right.$ DEPT and $\left.\mathrm{HMQC}\right)$ which was placed at C-28 instead of C-3 due to its interaction observed in the HMBC spectrum (Fig. 2) with C-28 and an $\alpha$ -
Table 2. ${ }^{13} \mathrm{C}-\mathrm{NMR}$ Data $(\delta / \mathrm{ppm})$ of $\mathbf{1}-\mathbf{3}^{a)}$

\begin{tabular}{|c|c|c|c|}
\hline Carbon & 1 & 2 & 3 \\
\hline 1 & 35.0 & 35.1 & 34.5 \\
\hline 2 & 27.7 & 28.0 & 27.9 \\
\hline 3 & 100.5 & 98.9 & 99.0 \\
\hline 4 & 38.5 & 38.5 & 38.5 \\
\hline 5 & 50.9 & 50.3 & 51.1 \\
\hline 6 & 19.7 & 19.6 & 19.6 \\
\hline 7 & 31.2 & 31.2 & 30.8 \\
\hline 8 & 40.7 & 40.3 & 43.7 \\
\hline 9 & 41.9 & 41.9 & 55.5 \\
\hline 10 & 34.7 & 35.0 & 35.1 \\
\hline 11 & 23.8 & 23.1 & 198.4 \\
\hline 12 & 125.9 & 126.1 & 127.9 \\
\hline 13 & 138.2 & 137.3 & 168.7 \\
\hline 14 & 42.1 & 42.5 & 44.0 \\
\hline 15 & 29.7 & 29.4 & 29.7 \\
\hline 16 & 24.9 & 24.9 & 23.7 \\
\hline 17 & 51.4 & 51.8 & 50.4 \\
\hline 18 & 49.3 & 49.4 & 39.5 \\
\hline 19 & 39.0 & 39.3 & 44.3 \\
\hline 20 & 38.5 & 38.7 & 30.1 \\
\hline 21 & 34.6 & 34.9 & 37.6 \\
\hline 22 & 75.3 & 75.7 & 75.3 \\
\hline 23 & 27.2 & 27.2 & 27.4 \\
\hline 24 & 16.9 & 16.9 & 17.7 \\
\hline 25 & 67.7 & 67.9 & 65.7 \\
\hline 26 & 18.4 & 18.4 & 18.9 \\
\hline 27 & 23.4 & 23.1 & 23.2 \\
\hline 28 & 178.9 & 178.0 & 178.1 \\
\hline 29 & 17.5 & 17.7 & 33.6 \\
\hline 30 & 21.0 & 21.2 & 25.9 \\
\hline $1^{\prime}$ & 165.3 & 170.0 & 166.3 \\
\hline $2^{\prime}$ & 116.0 & 20.7 & 127.6 \\
\hline $3^{\prime}$ & 156.8 & - & 138.8 \\
\hline $4^{\prime}$ & 27.4 & - & 15.6 \\
\hline $5^{\prime}$ & 20.2 & - & 20.4 \\
\hline $3-\mathrm{OCH}_{3}$ & 49.3 & - & - \\
\hline $28-\mathrm{OCH}_{3}$ & - & 51.2 & - \\
\hline
\end{tabular}

a) $100 \mathrm{MHz}$ in $\mathrm{CDCl}_{3} . \delta$ in ppm from TMS.

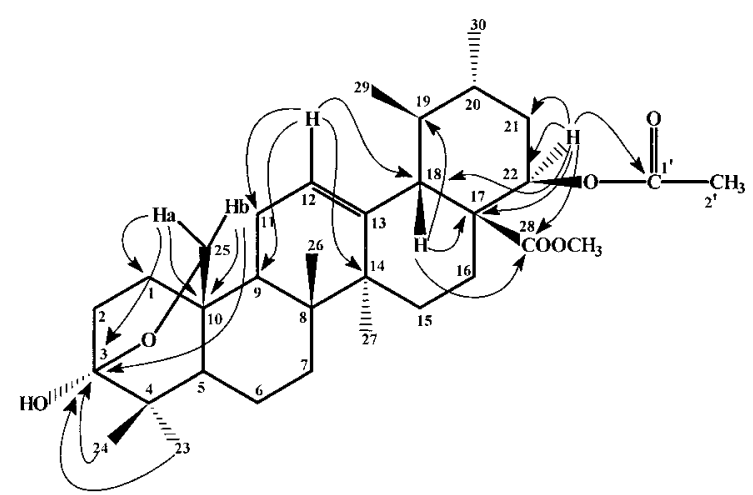

Fig. 2. Significant HMBC Interactions of 2

oriented $\mathrm{OH}$ group $\left(3450 \mathrm{~cm}^{-1}\right)$ was located at C-3 instead of $\mathrm{OMe}$. In the light of these observations, the structure of $\mathbf{2}$ has been elucidated as methyl $22 \beta$-acetoxy-3,25-epoxy- $3 \alpha$-hydroxy-urs-12-en-28-oate. Compound $\mathbf{2}$ is a new natural product, however, its synthesis from camarinic acid has been reported earlier. ${ }^{10)}$

Compound 3 showed a molecular ion peak $[\mathrm{M}]^{+}$at $\mathrm{m} / \mathrm{z}$ 582 in its EI-MS, the exact mass measurement (582.3546) of which gave its molecular formula as $\mathrm{C}_{35} \mathrm{H}_{50} \mathrm{O}_{7}$. Its IR spec- 


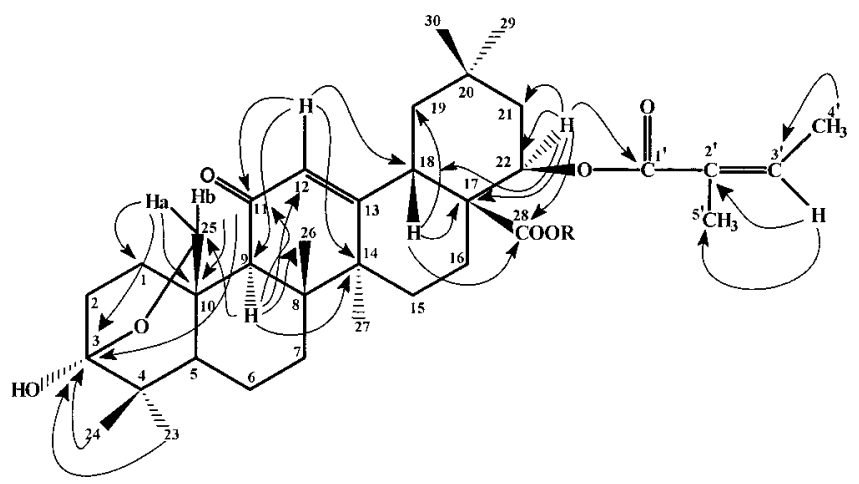

Fig. 3. Significant $\mathrm{HMBC}$ Interactions of $\mathbf{3} \mathrm{R}=\mathrm{H}$ and $\mathbf{3 a} \mathrm{R}=\mathrm{CH}_{3}$

trum showed absorption bands at 3450-2650 (br, OH, $\mathrm{COOH}), 2940,2850(\mathrm{CH}), 1730-1700$ (acid and ester $\mathrm{C}=\mathrm{O}), 1690(\alpha, \beta$ unsaturated ketone $)$ and $1620(\mathrm{C}=\mathrm{C})$. Its ultraviolet (UV) spectrum showed the absorption maximum at $248 \mathrm{~nm}$. The ${ }^{1} \mathrm{H}-\mathrm{NMR}$ spectrum (Table 1) showed six three-proton singlets at $\delta_{\mathrm{H}} 0.71,0.88,0.90,1.02,1.14$ and 1.36 , attributable to six tertiary methyls, a one-proton double doublet at $\delta_{\mathrm{H}} 3.20(J=14.0,4.2 \mathrm{~Hz})$ characteristic ${ }^{14)}$ of H-18 of $\Delta^{12}$ oleanene triterpenoids. A $\beta$-oriented hemiketal system at $\mathrm{C}-3$ with $\mathrm{C}-25$ was also indicated in compound $3\left[\delta_{\mathrm{H}} 4.50\right.$ $(1 \mathrm{H}, \mathrm{dd}, J=8.3,2.2 \mathrm{~Hz}, \mathrm{H}-25 \mathrm{a})$ and $\delta_{\mathrm{H}} 4.02(1 \mathrm{H}, \mathrm{dd}, J=8.3$, $1.1 \mathrm{~Hz}, \mathrm{H}-25 \mathrm{~b})]$. It also has a $\beta$-oriented ester side chain at $\mathrm{C}-22\left[\delta_{\mathrm{H}} 5.12(1 \mathrm{H}, \mathrm{t}, J=3.0 \mathrm{~Hz}) ; \delta_{\mathrm{C}} 75.3, \mathrm{HMQC}\right.$ spectrum]. The ${ }^{1} \mathrm{H}-\mathrm{NMR}\left[\delta_{\mathrm{H}} 6.01\left(1 \mathrm{H}, \mathrm{qq}, J=7.2,1.5 \mathrm{~Hz}, \mathrm{H}-3^{\prime}\right) ; \delta_{\mathrm{H}}\right.$ $1.94\left(1 \mathrm{H}, \mathrm{dq}, J=7.2,1.5 \mathrm{~Hz}, \mathrm{H}-4^{\prime}\right) ; \delta_{\mathrm{H}} 1.79(1 \mathrm{H}$, quintet, $\left.\left.J=1.5 \mathrm{~Hz}, \mathrm{H}-5^{\prime}\right)\right]$ and ${ }^{13} \mathrm{C}-\mathrm{NMR}$ spectral data (Table 2) and their correlations in the HMQC spectrum and HMBC connectivities (Fig. 3) indicated the presence of a 2,3-dimethylacrylic acid ester side chain with $Z$-configuration ${ }^{17)}$ which was further confirmed by interaction of $\mathrm{H}^{-3^{\prime}}\left(\delta_{\mathrm{H}} 6.01\right)$ with $5^{\prime}$-Me $\left(\delta_{\mathrm{H}} 1.79\right)$ in the NOESY spectrum. The $\mathrm{COOH}$ group indicated in the IR spectrum and confirmed by methylation with diazomethane $\left(\mathbf{3 a}, \delta_{\mathrm{H}}, 3.51, \mathrm{~s}\right)$ was located at $\mathrm{C}-17$ by comparing the ${ }^{13} \mathrm{C}$-NMR values of rings $\mathrm{C}, \mathrm{D}$ and $\mathrm{E}$ with those of similar compounds ${ }^{14)}$ as discussed for compound $\mathbf{1}$. These data showed a close similarity of $\mathbf{3}$ with that of camaric acid. ${ }^{14)}$ The compound also has an $\alpha, \beta$-unsaturated ketonic functionality $\left(1690 \mathrm{~cm}^{-1}, \lambda_{\max } 248 \mathrm{~nm}\right)$ which was placed at $\mathrm{C}-11$ due to the presence of a one-proton singlet at $\delta 5.74(\mathrm{H}-12)$ and ${ }^{13} \mathrm{C}-\mathrm{NMR}$ signals ${ }^{18)}$ at $\delta_{\mathrm{C}} 198.4(\mathrm{C}-11)$, 127.9 (C-12) and 168.7 (C-13). Connectivities of H-9 with C-11, C-12, C-14, C-25 and C-26 and of H-12 with C-9, C$11, \mathrm{C}-14$ and $\mathrm{C}-18$ supported this assignment. The remaining oxygen was placed at C-3 as $\alpha$-oriented hydroxy group as there is no further carbinylic proton in the NMR spectra. Thus the structure of $\mathbf{3}$ was established as 3,25-epoxy- $3 \alpha$-hydroxy-22 $\beta$-[(Z)-2'-methyl-2'-butenoyloxy]-11-oxo-olean-12en-28-oic acid.

\section{Experimental}

General The IR spectra were recorded on a JASCO A-302 spectrophotometer. The UV spectra were obtained on a HITACHI-U-3200 spectrophotometer. The EIMS and HREI-MS were recorded on Finnigan MAT-112 and JMS HX-110 spectrometers, respectively. The ${ }^{1} \mathrm{H}-\mathrm{NMR}$ spectra were recorded on a Bruker AM-400 FT-NMR spectrometer operating at $400 \mathrm{MHz}$, while the ${ }^{13} \mathrm{C}$-NMR spectra were obtained on the same instrument operating at $100 \mathrm{MHz}$. The chemical shifts are reported in $\delta(\mathrm{ppm})$, and the coupling constants are in $\mathrm{Hz}$. HMQC, ${ }^{1} \mathrm{H}-{ }^{1} \mathrm{H}$ COSY, NOESY and HMBC were obtained with the usual pulse sequence, and data processing was performed with Xwin-NMR software version 3.0. Silica gel 9385 (Merck) was used for column chromatography (CC) and flash column chromatography (FCC), and Silica gel $60 \mathrm{PF}_{254}$ (Merck) was used for vacuum liquid chromatography (VLC) and thick layer chromatography (TLC).

Plant Material The plant material was collected from the Karachi region in February 1997, and identified as L. camara by Mr. Abdul Ghafoor, Department of Botany, University of Karachi. A voucher specimen (No. $63482 \mathrm{KUH}$ ) has been deposited in the herbarium of the same university.

Extraction and Isolation Air-dried aerial parts of L. camara $(10 \mathrm{~kg})$ were repeatedly extracted with $\mathrm{MeOH}$ at room temperature. The concentrated extract, obtained on removal of the solvent from the combined extract under reduced pressure was partitioned between EtOAc and $\mathrm{H}_{2} \mathrm{O}$. The EtOAc phase was treated with $4 \% \mathrm{Na}_{2} \mathrm{CO}_{3}$ solution to separate the acidic from the neutral fraction. The EtOAc layer containing the neutral fraction was washed with water, dried $\left(\mathrm{Na}_{2} \mathrm{SO}_{4}\right)$, and passed through active charcoal. The charcoal bed was successively washed with EtOAc and $\mathrm{MeOH}-\mathrm{C}_{6} \mathrm{H}_{6}$ $(1: 1)$, which were combined on the basis of TLC. The residue obtained on removal of the solvent from the charcoal filtrates and washings was divided into hexane-soluble and hexane-insoluble fractions. The hexane-insoluble fraction was again divided into $\mathrm{Et}_{2} \mathrm{O}$-soluble and $\mathrm{Et}_{2} \mathrm{O}$-insoluble portions. The $\mathrm{Et}_{2} \mathrm{O}$-insoluble fraction was again divided into EtOAc-soluble and EtOAc-insoluble portions. The residue $(40 \mathrm{~g})$ obtained from the EtOAc-soluble portion was subjected to vacuum liquid chromatography (VLC) $\left(\mathrm{CHCl}_{3}\right.$; $\mathrm{CHCl}_{3}-\mathrm{MeOH}$ in order of increasing polarity), which yielded 9 fractions $(1-9)$. Fraction 6 obtained on elution with $\mathrm{CHCl}_{3}-\mathrm{MeOH}(9.5: 0.5)$ yielded pure $4(35.3 \mathrm{mg})$ as colourless crystallizate. Fraction $1(26 \mathrm{~g})\left(\mathrm{CHCl}_{3}\right.$; $\mathrm{CHCl}_{3}-\mathrm{MeOH}, 9.9: 0.1$ eluate) was further subjected to VLC (hexane, hexane-EtOAc, in order of increasing polarity), which ultimately furnished 8 fractions (I to VIII). Fr. II ( $216 \mathrm{mg}$ ) (hexane eluate) was subjected to flash column chromatography (FCC) (hexane, hexane-EtOAc, in order of increasing polarity) which furnished 9 fractions (II-1 to II-9). Fraction II-2 (hexane eluate) on purification through TLC $\left(\mathrm{CHCl}_{3}-\mathrm{MeOH}, 9.5: 0.5\right)$ gave 5 $(5.2 \mathrm{mg})$. Fraction II-3 (hexane eluate) afforded $6(16.5 \mathrm{mg})$. Fraction II-6 obtained on elution with hexane-EtOAc $(9: 1)$ yielded $7(3.3 \mathrm{mg})$.

Fraction III (2.6g) (hexane-EtOAc, $8: 2$ eluate) was subjected to FCC (hexane, hexane-EtOAc, in order of increasing polarity), which furnished 18 fractions (III-1 to III-18). Fraction III-14 obtained on elution with hexane-EtOAc $(9: 1)$ gave 1 (9.8 mg). Fraction III-17 (hexane-EtOAc, 9: 1 eluate) gave two major spots on TLC which on separation through TLC $\left(\mathrm{CHCl}_{3}-\mathrm{MeOH}, 9.5: 0.5\right)$ afforded $8(4 \mathrm{mg})$ and $9(6 \mathrm{mg})$. The main fraction $3(4.7 \mathrm{~g})\left(\mathrm{CHCl}_{3}-\mathrm{MeOH}, 9.8: 0.2\right.$ eluate) was further subjected to VLC $\left(\mathrm{CHCl}_{3} ; \mathrm{CHCl}_{3}-\mathrm{MeOH}\right.$ in order of increasing polarity), which yielded 9 fractions (3-1 to 3-9). Fraction 3-3 $(600 \mathrm{mg})\left(\mathrm{CHCl}_{3}\right.$ eluate) was subjected to flash column chromatography (FCC) (hexane, hexane-EtOAc in order of increasing polarity) which furnished 7 fractions (3-3-I to 3-3-VII). Fraction 33 -VI obtained on elution with hexane-EtOAc $(9: 1)$ afforded pure 2 $(58.1 \mathrm{mg})$. Fraction 3-3-IV $(269.5 \mathrm{mg})$ was subjected to column chromatography (CC) $\left(\mathrm{CHCl}_{3} ; \mathrm{CHCl}_{3}-\mathrm{MeOH}\right.$, in order of increasing polarity) which furnished 12 fractions (3-3-IV-1 to 3-3-IV-12). Fraction 3-3-IV-10 obtained on elution with $\mathrm{CHCl}_{3}-\mathrm{MEOH}(9.8: 0.2)$ afforded $3(10.0 \mathrm{mg})$.

Camaryolic acid (1): An amorphous powder; $[\alpha]_{\mathrm{D}}+169^{\circ}(c=0.10$, $\left.\mathrm{CHCl}_{3}\right)$; UV $\lambda_{\max }(\mathrm{MeOH}) \mathrm{nm}: 217$; IR $\left(\mathrm{CHCl}_{3}\right) \mathrm{cm}^{-1}: 3450-2620(\mathrm{br}$, $\mathrm{COOH}), 1730($ ester $\mathrm{C}=\mathrm{O}), 1710($ acid $\mathrm{C}=\mathrm{O}), 1620(\mathrm{C}=\mathrm{C})$; EI-MS $\mathrm{m} / \mathrm{z}$ (rel. int. \%): $582[\mathrm{M}]^{+}$(7), 482 (18), 285 (4), 249 (10), 246 (7), 236 (9), 201 (6), 119 (40), 83 (100), 55 (65); HR-EI-MS m/z: 582.3918 (Calcd for $\left.\mathrm{C}_{36} \mathrm{H}_{54} \mathrm{O}_{6}, 582.3920\right), 482.3382\left(\mathrm{C}_{31} \mathrm{H}_{46} \mathrm{O}_{4}\right), 285.1859\left(\mathrm{C}_{19} \mathrm{H}_{25} \mathrm{O}_{2}\right), 249.1850$ $\left(\mathrm{C}_{16} \mathrm{H}_{25} \mathrm{O}_{2}\right), 246.1615\left(\mathrm{C}_{16} \mathrm{H}_{22} \mathrm{O}_{2}\right), 236.1770\left(\mathrm{C}_{15} \mathrm{H}_{24} \mathrm{O}_{2}\right), 201.1650\left(\mathrm{C}_{15} \mathrm{H}_{21}\right)$, $119.0864\left(\mathrm{C}_{9} \mathrm{H}_{11}\right), 83.0484\left(\mathrm{C}_{5} \mathrm{H}_{7} \mathrm{O}\right) ;{ }^{1} \mathrm{H}$ - and ${ }^{13} \mathrm{C}$-NMR: see Tables 1 and 2.

Methylation of 1 Compound $\mathbf{1}(5 \mathrm{mg})$ formed methyl derivative 1a $(4.5 \mathrm{mg})$ on treatment with an ethereal solution of diazomethane and the usual workup. EI-MS m/z: $596\left[\mathrm{M}^{+}\right]$; ${ }^{1} \mathrm{H}-\mathrm{NMR}\left(\mathrm{CDCl}_{3}\right) \delta: 0.72(3 \mathrm{H}, \mathrm{s}, \mathrm{H}-$ 24), $0.84(3 \mathrm{H}, \mathrm{d}, J=6.0 \mathrm{~Hz}, \mathrm{H}-29), 0.88(3 \mathrm{H}, \mathrm{d}, J=6.2 \mathrm{~Hz}, \mathrm{H}-30), 0.93(3 \mathrm{H}$, $\mathrm{s}, \mathrm{H}-26), 1.00(3 \mathrm{H}, \mathrm{s}, \mathrm{H}-23), 1.06(3 \mathrm{H}, \mathrm{s}, \mathrm{H}-27), 1.80\left(3 \mathrm{H}, \mathrm{s}, \mathrm{H}-4^{\prime}\right), 2.00$ $\left(3 \mathrm{H}, \mathrm{s}, \mathrm{H}-5^{\prime}\right), 2.41(1 \mathrm{H}, \mathrm{d}, J=11.0 \mathrm{~Hz}, \mathrm{H}-18), 3.24(3 \mathrm{H}, \mathrm{s}, 3-\mathrm{OMe}) 3.50(3 \mathrm{H}$, $\mathrm{s}$, COOMe $), 3.85(1 \mathrm{H}, \mathrm{dd}, J=8.8,1.0 \mathrm{~Hz}, \mathrm{H}-25 \mathrm{~b}), 4.25(1 \mathrm{H}, \mathrm{dd}, J=8.8$, $2.6 \mathrm{~Hz}, \mathrm{H}-25 \mathrm{a}), 5.01(1 \mathrm{H}, \mathrm{t}, J=3.2 \mathrm{~Hz}, \mathrm{H}-22 \alpha), 5.20(1 \mathrm{H}, \mathrm{t}, J=3.5 \mathrm{~Hz}, \mathrm{H}-$ 12), $5.53\left(1 \mathrm{H}, \mathrm{s}, \mathrm{H}-2^{\prime}\right)$.

Methylcamaralate (2): An amorphous powder; $[\alpha]_{\mathrm{D}}+171^{\circ}(c=0.12$, $\left.\mathrm{CHCl}_{3}\right)$; UV $\lambda_{\text {max }}(\mathrm{MeOH}) \mathrm{nm} ; 210$; IR $\left(\mathrm{CHCl}_{3}\right) \mathrm{cm}^{-1}: 3450(\mathrm{OH}), 1740$ $($ ester $\mathrm{C}=\mathrm{O}), 1620(\mathrm{C}=\mathrm{C})$; EI-MS $m / z$ (rel. int. \%): $542[\mathrm{M}]^{+}(2), 482(17)$, 299 (5), 260 (8), 201 (38), 185 (16), 133 (17), 119 (53), 105 (19), 69 (100); HR-EI-MS m/z: 542.3900 (Calcd for $\mathrm{C}_{33} \mathrm{H}_{50} \mathrm{O}_{6}, 542.3912$ ), 482.3389 
$\left(\mathrm{C}_{31} \mathrm{H}_{46} \mathrm{O}_{4}\right), 299.2014\left(\mathrm{C}_{20} \mathrm{H}_{27} \mathrm{O}_{2}\right), 260.1774\left(\mathrm{C}_{17} \mathrm{H}_{24} \mathrm{O}_{2}\right), 201.1638\left(\mathrm{C}_{15} \mathrm{H}_{21}\right)$, $185.1328\left(\mathrm{C}_{14} \mathrm{H}_{17}\right), 133.1015\left(\mathrm{C}_{10} \mathrm{H}_{13}\right), 119.0858\left(\mathrm{C}_{9} \mathrm{H}_{11}\right), 105.0702\left(\mathrm{C}_{8} \mathrm{H}_{9}\right)$, $69.0701\left(\mathrm{C}_{5} \mathrm{H}_{9}\right) ;{ }^{1} \mathrm{H}$ - and ${ }^{13} \mathrm{C}$-NMR: see Tables 1 and 2 .

Camangeloyl acid (3): An amorphous powder; $[\alpha]_{\mathrm{D}}+165^{\circ}(c=0.15$, $\left.\mathrm{CHCl}_{3}\right)$; UV $\lambda_{\max }(\mathrm{MeOH}) \mathrm{nm}: 248$; IR $\left(\mathrm{CHCl}_{3}\right) \mathrm{cm}^{-1}: 3450-2650$ (br, OH, $\mathrm{COOH}), 2940,2850(\mathrm{CH}), 1730-1700$ (acid and ester $\mathrm{C}=\mathrm{O}), 1690(\alpha, \beta$ unsaturated ketone), $1620(\mathrm{C}=\mathrm{C})$; EI-MS $m / z$ (rel. int. \%): $582[\mathrm{M}]^{+}(2)$, 564 (17), 519 (96), 482 (4), 466 (15), 419 (98), 260 (4), 242 (3), 185 (16), 133 (30), 119 (53), 105 (40), 55 (100); HR-EI-MS m/z: 582.3546 (Calcd for $\left.\mathrm{C}_{35} \mathrm{H}_{50} \mathrm{O}_{7}, 582.3556\right), 564.3448\left(\mathrm{C}_{35} \mathrm{H}_{48} \mathrm{O}_{6}\right), 519.3470\left(\mathrm{C}_{34} \mathrm{H}_{47} \mathrm{O}_{4}\right), 482.3028$ $\left(\mathrm{C}_{30} \mathrm{H}_{42} \mathrm{O}_{5}\right), 466.3078\left(\mathrm{C}_{30} \mathrm{H}_{42} \mathrm{O}_{4}\right), 419.2945\left(\mathrm{C}_{29} \mathrm{H}_{39} \mathrm{O}_{2}\right), 260.1410\left(\mathrm{C}_{16} \mathrm{H}_{20} \mathrm{O}_{3}\right)$, $242.1301\left(\mathrm{C}_{16} \mathrm{H}_{18} \mathrm{O}_{2}\right), 185.1329\left(\mathrm{C}_{14} \mathrm{H}_{17}\right), 133.1018\left(\mathrm{C}_{10} \mathrm{H}_{13}\right), 119.0859$ $\left(\mathrm{C}_{9} \mathrm{H}_{11}\right), 105.0706\left(\mathrm{C}_{8} \mathrm{H}_{9}\right) ;{ }^{1} \mathrm{H}$ - and ${ }^{13} \mathrm{C}-\mathrm{NMR}$ : see Tables 1 and 2 .

Methylation of 3 Compound $3(8 \mathrm{mg})$ afforded methyl derivative 3a $(7.0 \mathrm{mg})$ on treatment with an ethereal solution of diazomethane and the usual workup. EI-MS $m / z: 596\left[\mathrm{M}^{+}\right] ;{ }^{1} \mathrm{H}-\mathrm{NMR}\left(\mathrm{CDCl}_{3}\right) \delta: 0.72(3 \mathrm{H}, \mathrm{s}, \mathrm{H}-$ 24), 0.87 (3H, s, H-29), 0.92 (3H, s, H-26), $1.02(3 \mathrm{H}, \mathrm{s}, \mathrm{H}-23), 1.12$ (3H, s, H-30), 1.34 (3H, s, H-27), 1.78 ( $3 \mathrm{H}$, quintet, $\left.J=1.4 \mathrm{~Hz}, \mathrm{H}-5^{\prime}\right), 1.94$ ( $3 \mathrm{H}, \mathrm{dq}$, $\left.J=7.0,1.4 \mathrm{~Hz}, \mathrm{H}-4^{\prime}\right), 3.22(1 \mathrm{H}, \mathrm{dd}, J=14.2,4.1 \mathrm{~Hz}, \mathrm{H}-18), 3.51(3 \mathrm{H}, \mathrm{s}$, COOMe $), 4.01(1 \mathrm{H}, \mathrm{dd}, J=8.2,1.0 \mathrm{~Hz}, \mathrm{H}-25 \mathrm{~b}), 4.51(1 \mathrm{H}, \mathrm{dd}, J=8.2,2.0 \mathrm{~Hz}$, $\mathrm{H}-25 \mathrm{a}), 5.13(1 \mathrm{H}, \mathrm{t}, J=3.2 \mathrm{~Hz}, \mathrm{H}-22 \alpha), 5.73(1 \mathrm{H}, \mathrm{s}, \mathrm{H}-12), 6.00(1 \mathrm{H}, \mathrm{qq}$, $\left.J=7.0,1.4 \mathrm{~Hz}, \mathrm{H}-3^{\prime}\right)$.

\section{References and Notes}

1) Kirtikar K. R., Basu B. D., "Indian Medicinal Plants," ed. by Basu S. N., Panini Office, Bhuwaneswari Asrama, Bahadurganj: Allahabad, India, 1981, pp. 984-986.

2) Sastri B. N., "The Wealth of India," Vol. VI, Council of Scientific and Industrial Research, New Delhi, 1962, pp. 31-34.
3) Sharma O. P., Dawra R. K., Ramesh D., Phytochemistry, 29, 39613962 (1990).

4) Sharma V. N., Kaul K. N., Br. Patent No. 8205211959.

5) Rwangabo P. C., Claeys, M., Pieters L., Corthout J., Vanden Berghe D. A., Vlietink A. J., J. Nat. Prod., 51, 966-968 (1988).

6) Irbarren A. M., Pomilio A. B., J. Nat. Prod., 46, $752-753$ (1983).

7) Gunstone F. D., Chem. Phys. Lipids, 17, $1-3$ (1976).

8) Joshi U. R., Kapadi A. H., Synth. Commun., 14, 681-686 (1984).

9) Bailey A. V., J. Am. Oil Chem. Soc., 48, 775-777 (1971).

10) Siddiqui B. S., Raza S. M., Begum S., Siddiqui S., Phytochemistry, 38, 681-685 (1995).

11) Barua A. K., Chakrabarti P., Dutta S. P., Mukherjee D. K., Tetrahedron, 27, 1141-1147 (1971).

12) Seo S., Tomita Y., Tori K., J. Am. Chem. Soc., 103, 2075-2080 (1981).

13) Budzikiewicz H., Wilson J. M., Djerassi C., J. Am. Chem. Soc., 85, 3688-3699 (1963).

14) Begum S., Raza S. M., Siddiqui B. S., Siddiqui S., J. Nat. Prod., 58, 1570-1574 (1995).

15) Miana G. A., Al-Hazimi H. M. G., Phytochemistry, 26, 225-227 (1987).

16) Barua A. K., Chakrabarti P., Chowdhury M. K., Basak K., Basu K., Ray S., Shaha S. K., J. Indian Chem. Soc., 62, 298-305 (1985).

17) Cerda-Garcia-Rojas C. M., Sanchez-Arreola E., Joesph-Nathan P., Roman L. U., Hernandez J. D., Phytochemistry, 32, 1219-1223 (1993).

18) Begum S., Wahab A., Siddiqui B. S., Qamar F., J. Nat. Prod., 63, 765-767 (2000). 